\title{
Toenail as a biomarker of heavy metal exposure via drinking water: a systematic review
}

\begin{abstract}
Toenail is metabolic end product of the skin, which can provide information about heavy metal accumulation in human cells. Slow growth rates of toenail can represent heavy metal exposure from 2 to 12 months before the clipping. The toenail is a non-invasive biomarker that is easy to collect and store and is stable over time. In this systematic review, the suitability of toenail as a long-term biomarker was reviewed, along with the analysis and validation of toenail and confounders to heavy metal. This systematic review has included 30 articles chosen from a total of 132 articles searched from online electronic databases like Pubmed, Proquest, Science Direct, and SCOPUS. Keywords used in the search included "toenail", "biomarker", "heavy metal", and "drinking water". Heavy metal in toenail can be accurately analyzed using an ICP-MS instrument. The validation of toenail heavy metal concentration data is very crucial; however, the Certified Reference Material (CRM) for toenail is still unavailable. Usually, CRM for hair is used in toenail studies. Confounders that have major effects on heavy metal accumulation in toenail are dietary intake of food and supplement, smoking habit, and overall health condition. This review has identified the advantages and limitations of using toenail as a biomarker for long-term exposure, which can help future researchers design a study on heavy metal exposure using toenail.
\end{abstract}

Keyword: Confounders; Drinking water; Heavy metal; Toenail 